\section{Research Square}

\title{
Ferroptosis Regulators and Tumor Microenvironment Immune Cell Infiltration Characterization in Adrenocortical Carcinoma
}

\section{Chengquan Shen ( $\nabla$ doctorchengquan@sina.com )}

The Affiliated Hospital of Qingdao University https://orcid.org/0000-0001-8402-2271

Jing Liu

The Affiliated Hospital of Qingdao University

Ye Liang

The Affiliated Hospital of Qingdao University

Zhijuan Liang

The Affiliated Hospital of Qingdao University

\section{Liping Wang}

The Affiliated Hospital of Qingdao University

\section{Yuanbin Chen}

The Affiliated Hospital of Qingdao University

\section{Dan Li}

The Affiliated Hospital of Qingdao University

\section{Yonghua Wang}

The Affiliated Hospital of Qingdao University

\section{Research Article}

Keywords: Adrenocortical carcinoma, Ferroptosis, Tumor Microenvironment Immune Cell, Prognosis

Posted Date: January 11th, 2022

DOI: https://doi.org/10.21203/rs.3.rs-1242199/v1

License: (c) (1) This work is licensed under a Creative Commons Attribution 4.0 International License. Read Full License 


\section{Abstract \\ Background}

Adrenocortical carcinoma (ACC) is a rare disease with a poor prognosis and lacking effective systemic treatment options. Recent studies showed that ferroptosis play a prominent role in the initiation and development of cancer. Nonetheless, the potential roles of ferroptosis regulators in the prognosis and tumor microenvironment immunomodulator factors expression remain not fully study.

\section{Methods}

TCGA and GEO ACC datasets were used to investigate the relationship between ferroptosis regulators with prognosis and clinical features. Consensus clustering analysis was performed to divided ACC patients into different ferroptosis subgroups. A ferroptosis scoring system was established for individual ACC using principal component analysis algorithms. The correlation between ferroptosis score and tumor microenvironment immune cell infiltration was analyzed.

\section{Results}

Twenty ferroptosis regulators were differentially expressed in ACC and 17 ferroptosis regulators were closely related to the prognosis of ACC. Three ferroptosis subgroups (Cluster A, B, and C) were determined based on the expression of ferroptosis regulators. Cluster $\mathrm{C}$ is preferentially associated with favorable OS, PFS, upregulated antigen-presenting genes expression, and higher immune cell infiltration. GSEA also indicated that Cluster $\mathrm{C}$ was prominently related to immune fully activation including chemokine signaling pathway, natural killer cell-mediated cytotoxicity, T cell receptor signaling pathway, and Toll-like receptor signaling pathway. A ferroptosis scoring system was constructed and it could serve as an independent prognostic factor for ACC. The ferroptosis scores were significantly correlated with TMB, immune-checkpoint genes expression, and tumor microenvironment immune cell infiltration in ACC. Further analyses indicated that the ferroptosis score integrated with TMB, immune-checkpoint genes expression, and CD4+ T cell infiltration, could predict the prognosis of ACC. Furthermore, a nomogram was constructed to monitor the prognosis of individual ACC patient. RNA isolation and reverse transcription-quantitative PCR (RT-qPCR) demonstrated significant differences in the expression levels of ACSL4, FANCD2 and SLC7A1 between ACC and normal tissues.

\section{Conclusion}

Our study demonstrated ferroptosis regulators were significantly associated with the prognosis, clinical characteristics, immune-checkpoint genes expression, and tumor microenvironment immune cell infiltration in ACC. This current study provided comprehensive evidence for further research on ferroptosis 
regulators in ACC and provides new enlightenment for epigenetic regulation of antitumor immune response.

\section{Background}

Adrenocortical carcinoma (ACC) is a rare and highly aggressive disease for which diagnostic approaches and therapeutic strategies have only gradually changed over the past decades [1-2]. Currently, surgical resection is the main treatment modality to prolong the prognosis of early $\mathrm{ACC}$, but for advanced or metastatic ACC, mitotane, chemotherapy, or other traditional adjuvant treatments often have difficulty achieving satisfactory therapeutic effect due to the heterogeneity of the ACC tumor microenvironment. Recent discoveries indicated that ferroptosis plays a vital role in the depression of cancer growth and proliferation. Ferroptosis is a new iron-dependent and reactive oxygen species form of regulated cell death that is morphologically, biochemically, and genetically different from other types of cell death such as apoptosis, necroptosis, autophagy, and pyroptosis [3]. Emerging evidence implicated that ferroptosis may be an adaptive process, which was essential in cancer cells eradication [4] and its activation enhances the therapeutic efficacy of anticancer agents [5]. Importantly, increasing evidence suggests that ferroptosis influences therapeutic resistance and evasion of immune surveillance and by remodeling its immune microenvironment and modulating metabolic pathway [6]. Dai et al. reported that autophagydependent ferroptosis is essential for the polarization of tumor-associated macrophages, which are oriented toward promoting tumor growth, remodeling tumor microenvironment, and suppressing tumor immunity [7].

Additionally, Wang et al. found that immunotherapy-activated CD8+ T cells enhance tumor cell ferroptosis, which in turn promote the anti-tumor efficacy of immunotherapy [8]. Meanwhile, tumorinfiltrating immune cells and immune checkpoint inhibitor therapy also have prominent roles in ACC [910]. Thus, it is particularly important to further study the interaction between ferroptosis regulators and immune molecules in the tumor microenvironment, which regulate the function of both tumor cells and immune cells, and then identify the factors closely related to the prognosis of patients with ACC.

In our study, we first identified the relationship between ferroptosis regulators and the prognosis of ACC patients. Moreover, we further explored the relationship between ferroptosis score and immune cell infiltration in the ACC microenvironment, and construct a nomogram to predict the prognosis of individual ACC patients by integrating with the clinical feature, immune-checkpoint genes expression, CD $4+T$ cell infiltration level, and ferroptosis score.

\section{Methods}

\subsection{Data collection}

The RNA sequencing transcriptome data of ACC patients and the corresponding clinical data were extracted from TCGA (https://tcga-data.nci.nih.gov/tcga/) and GEO (https://www. ncbi.nlm.nih.gov/geo/, 
GSE19776,) databases. The RNA sequencing transcriptome data of normal samples download from the Genotype-Tissue Expression (GTEx) database (https://xenabrowser.net/datapages/). One hundred and twenty-seven ACC samples (TCGA: 79; GES19776: 48) and 155 normal samples were enrolled in our study. Twenty-four ferroptosis regulators (CDKN1A, HSPA5, EMC2, SLC7A1, MT1G, HSPB1, FANCD2, SLC1A5, RPL8, LPCAT3, DPP4, CARS, NFE2L2, GPX4, CISD1, FDFT1, NCOA4, GLS2, CS, ATP5MC3, ACSL4, SAT1, TFRC, ALOX15) were collected from previously published literature. Immune cell fraction data were obtained from Tumor Immune Estimation Resource (TIMER) website (http://cistrome.dfci.harvard.edu/TIMER/), which contained TIMER, CIBERSORT, EPIC, MCPCOUNTER, QUANTISEQ, and XCELL datasets.

\subsection{Consensus Clustering of ferroptosis regulators}

To functionally elucidate the biological characteristics of the ferroptosis regulators in ACC, consensus clustering analysis with "ConsensusClusterPlus" was employed to classify ACC patients into different groups based on the expression levels of twenty-four ferroptosis regulators. The principal component analysis (PCA) was performed to evaluate gene-expression profiles among distinct ACC subtypes.

\subsection{Single-sample gene-set enrichment analysis}

Single-sample gene-set enrichment analysis (SSGSEA) algorithm has been widely to quantify the relative abundance of immune cell infiltration in the tumor microenvironment. The gene set for marking each immune cell type was extracted from the study of Charoentong, which contained various immune cell types, such as Activated B cell, Activated CD4 T cell, Activated CD8 T cell, Activated dendritic cell, Macrophage and so on [11]. ssGSEA calculated the enrichment fractions, which were used represent the relative abundance of each immune cell infiltration level in the tumor microenvironment of each ACC sample.

\subsection{Gene set variation analysis}

Gene set variation analysis (GSVA) is usually used for determines biological process activity divergences in the samples of an expression dataset in a non-parametric and unsupervised manner [12]. To identify the difference in biological process between ferroptosis regulators related subgroups, gene set variation analysis (GSVA) enrichment analysis was performed by using "GSVA" R packages.

\subsection{Generation of a ferroptosis regulators related scoring system}

To quantify the ferroptosis level of an individual tumor, a ferroptosis regulators related scoring system was established to assess the ferroptosis level of individual ACC patients, and we termed it as a ferroptosis score. Univariate Cox regression analysis was performed on ferroptosis regulators to screen candidate genes that were closely related to the prognosis of patients with ACC. PCA was then conducted to establish a ferroptosis regulators related scoring system. The advantage of PCA is concentrating scores on the set with the largest block of highly relevant (or inversely relevant) genes in the set while reducing the contribution weight of genes that do not track with other set members [13]. 


\subsection{Construction of a prognostic nomogram}

Clinicopathological characteristics, TMB (tumor mutation burden), immune-checkpoint genes (PD-L1, programmed death-ligand 1; CTLA4, cytotoxic T-lymphocyte associated protein 4; PD-1, programmed death 1) expression, CD4+ T cell infiltration level, and ferroptosis score were integrated to construct a nomogram that was used to assess the probability of 1-, 2-, and 3-year overall survival (OS) and progress free survival (PFS) for ACC patients via the R package (https://cran.r-project.org/web/packages/rms/) [14].

\subsection{RNA Isolation and Reverse Transcription-Quantitative PCR}

To further validate the expression levels of three ferroptosis regulators that were utilized to construct the signature in our ACC tissues and normal tissues, RNA isolation and reverse transcription-quantitative PCR (RT-qPCR) were performed. The RNAprep pure FFPE kit (DP439, TIANGEN Biotech(Beijing)Co, Ltd, $\mathrm{CHN}$ ) was used to extract the total RNA from tissue specimens based on the manufacturer's instructions. For the detection of mRNA levels, the total RNA (500 ng) was transcribed into cDNA using a PrimeScript ${ }^{\text {TM }}$ RT reagent kit (Perfect Real Time) (Takara, code no RR037A). All the primers were synthesized by Huada Gene (Beijing, China) and the sequences are shown in Table 1. The amplification of cDNAs was conducted with Roche LightCycler 480II real-time PCR detection system (Roche, Basel, Switzerland). Gene expression was normalized against $\beta$-Actin and relative expression levels of ACSL4, FANCD2, and SLC7A1 were determined by the comparative threshold cycle $(\mathrm{Ct})$ method using the formula $2-(\Delta \Delta \mathrm{Ct})$.

Table 1

Sequences of the primers used for real-time quantitative PCR.

\begin{tabular}{|ll|}
\hline Name of primer & Sequence of primer $\mathbf{( 5}{ }^{\prime}$ to $\mathbf{3}^{\mathbf{}} \mathbf{)}$ \\
\hline ACSL4-F & GCTATCTCCTCAGACACACCGA \\
\hline ACSL4-R & AGGTGCTCCAACTCTGCCAGTA \\
\hline FANCD2-F & ACATACCTCGACTCATTGTCAGT \\
\hline FANCD2-R & TCGGAGGCTTGAAAGGACATC \\
\hline SLC7A1-F & GCCTGTGCTATGGCGAGTTT \\
\hline SLC7A1-R & ACGCTTGAAGTACCGATGATGTA \\
\hline B-Actin-F & GGCATCGTCACCAACTGGGAC \\
\hline B-Actin-R & CGATTTCCCGCTCGGCCGTGG \\
\hline
\end{tabular}

\subsection{Statistical analysis}


Statistical tests were performed using R version 3.6.0 and GraphPad Prism 8.0. The expression levels of ferroptosis regulators in ACC subtypes, and between tumor and normal tissues were compared using One-way ANOVA and t-test. Depending on the relationship between ferroptosis regulators or ferroptosis score and ACC patients' survival, the "survminer" R package was used to determine the cut-off point for each subgroup of the dataset. We use the Kaplan-Meier method to generate survival curves and compared the difference between groups with the log-rank test. The correlation between ferroptosis score and immune cell infiltration levels was subjected to using a Pearson correlation test in GraphPad Prism 8.0. Univariate and multivariate Cox regression analyses were performed to ascertain the independent prognostic value of the ferroptosis score integrated with other clinicopathological characteristics.

\section{Results}

\subsection{The landscape of genetic variation of ferroptosis regulators in ACC}

To explore the biological function of ferroptosis regulators in the occurrence and development of ACC, we systematically assess the expression profiles of 24 ferroptosis regulators between ACC and normal samples. Compared to the expression levels of ferroptosis regulators in normal samples, CDKN1A, HSPA5, SLC7A1, HSPB1, FANCD2, RPL8, DPP4, GPX4, FDFT1, NCOA4, and ACSL4 were dramatically upregulated in ACC samples, while EMC2, MT1G, SLC1A5, LPCAT3, CAR3, NFE2L2, GLS2, ATP5MC3, and STA1 were markedly down-regulated in ACC samples. However, the expression of CISD1, CS, TFRC, and ALOX15 were no statistically significant difference between normal and ACC samples (Figure. 1A-B). Meanwhile, we analyzed the incidence of somatic mutations of 24 ferroptosis regulators in ACC. Among 92 samples, only 6 experienced mutations of ferroptosis regulators, with a frequency of $6.52 \%$. SLC1A5 and SAT1 exhibited the highest mutation frequency followed by FDFT1 and CS, while other ferroptosis regulators show any mutations in ACC samples (Figure. 1C). We further analyze the difference in the expression of ferroptosis regulators between SLC1A5-mutant and wild types or SAT1-mutant and wild types. The results indicated that NCOA4 was highly expressed in SLC1A5-mutant types, while MT1G was lowly expressed in wild types (Figure. 1D-E, Supplementary 1). However, no ferroptosis regulators were differentially expressed between SAT1-mutant and wild types (Supplementary 2). The exploration of copy number variations (CNV) alteration frequency indicated a prevalent CNV alteration in 24 ferroptosis regulators and 19 regulators were focused on the amplification in copy number, while NCOA4, HSPB1, ALOX15, ACSL4 CISD1, and SLC7A1 had a widespread frequency of CNV deletion (Figure. 1F). Fig. 1Gshowed the position of CNV-altered ferroptosis regulators on chromosomes. These results suggested that the expression imbalance of ferroptosis regulators possessed critical biological roles in the progression of ACC.

\subsection{Prognostic values of ferroptosis regulators in ACC}

To further investigate the importance of ferroptosis regulators in ACC, survival analysis was performed to assess the prognostic value of each ferroptosis regulator using the "survminer" R package. The results 
showed that ACSL4, CDKN1A, CISD1, EMC2, FANCD2, FDFT1, GPX4, HSPA5, HSPB1, RPL8, SAT1, SLC1A5, SLC7A1, and TFRC were closely related to the OS and PFS of ACC. Moreover, CS and ATP5MC3 were related to the OS of ACC, and GLS2 and NFE2L2 were related to the PFS of ACC (Figure. 2A-B). Among these prognostic ferroptosis regulators, eight regulators were positively correlated with the prognosis of ACC, while nine regulators were negatively correlated with the prognosis of ACC. Furthermore, the relationship between prognostic ferroptosis regulators and clinical characteristics (Stage, T, N, and M) was analyzed. We found that patients with high stage had higher expression of FANCD2, SLC1A5, SLC7A1, and TFRC, but lower expression of ACSL4, ATP5MC3, EMC2, GPX4, and LPCAT3 (Figure. 3A). FANCD2, SLC7A1, and TFRC have high expression, while ATP5CM3 and EMC2 have low expression in ACC patients with advanced T (Figure. 3B). Compared to ACC patients without lymph node metastasis, HSPB1 down-regulated in those with lymph node metastasis (Figure. 3C). Moreover, FANCD2 and SLC7A1 may promote the metastasis of ACC, while EMC2 and HSPB1 may inhibit the metastasis of ACC (Figure. 3D). Overall, the expression of ferroptosis regulators not only was closely related to the prognosis of ACC but was significantly related to the clinical characteristics of ACC, which indicate the important roles of ferroptosis regulators in tumorigenesis and progression of ACC.

\subsection{Tumor environment immune cell infiltration characterization in distinct ferroptosis subgroups}

To investigate the biological of different functional groups of ferroptosis regulators, the ConsensusClusterPlus was used to classify ACC patients with qualitatively different ferroptosis subgroups based on the expression levels of ferroptosis regulators, and three distinct subgroups were eventually identified, namely, cluster $A(n=31)$, cluster $B(n=39)$, and cluster $C(n=57)$, respectively (Supplementary 3A-C). PCA analysis showed that a prominent distinction in three different ferroptosis subgroups (Supplementary 3D). Cluster A has six regulators that were overexpressed and were closely related to the poor prognosis of ACC. Cluster B present moderated expression in most differentially expressed regulators except for the HSPB1. Cluster $C$ has six regulators that were highly expressed, and most of them were related to the better clinical outcomes of ACC (Figure. 4A-B). Interestingly, survival analyses for three subgroups also revealed that patients in Cluster $C$ had significantly longer $O S(P<$ $0.001)$ and PFS $(P<0.001)$ than those in Cluster A or Cluster B (Figure. 4C-D ). Therefore, our results further suggested that ferroptosis regulators were significantly correlated with the heterogeneity of patients with ACC.

Furthermore, GSVA enrichment analysis was performed to explore the biological behaviors between different ferroptosis groups. The results showed that Cluster A markedly enrich in ECM receptor interaction, steroid biosynthesis, terpenoid backbone biosynthesis, cell cycle, and DNA replication (Figure. 4E). Cluster B presented enrichment pathways associated with steroid biosynthesis, terpenoid backbone biosynthesis, nucleotide excision repair, and cell cycle (Figure. 4F). Cluster $\mathrm{C}$ was prominently related to immune fully activation including the chemokine signaling pathway, natural killer cell-mediated cytotoxicity, $T$ cell receptor signaling pathway, and Toll-like receptor signaling pathway, and so on (Figure. $4 G$ ). In addition, Cluster $C$ also has a higher immune score than cluster A or Cluster B (Figure. $4 \mathrm{H}$ ). 
Subsequently, we analyzed the immune cell infiltration and antigen-presenting genes expression among different ferroptosis groups. Cluster $\mathrm{C}$ showed higher infiltration levels of immune cells in the sSGSEA dataset, such as activated B cell, activated CD4T cell, CD8T cell, macrophage, $T$ follicular helper cell, and so on (Figure. 4I). We then used other datasets (CIBERSORT, MCPCOUNT, QUANTISQ, TIMER, XCELL) to compare differences of immune cells among the three subgroups. We also found that there were significant differences in the compositions of immune cells types among the three subgroups, especially CD4+ $T$ cell, $C D 8+T$ cell, and macrophage (Figure. 4J-N).

Considering the important roles of antigen-presenting genes in the immune response of tumors, we assessed the expression of these genes in three ferroptosis subgroups. The results showed that most antigen-presenting genes were highly expressed in Cluster $C$, representing the strong immunogenicity of Cluster $\mathrm{C}$ in ACC (Figure. 40). Taken together, ferroptosis regulators have critical roles in antitumor immune response in ACC.

\subsection{Generation of a ferroptosis regulators related scoring system}

Three ferroptosis regulators were identified as candidate genes to generated a scoring system by using univariate Cox regression analysis. Subsequently, PCA was used to generate a ferroptosis score for each ACC patient based on the expression values of ferroptosis regulators. Afterward, patients were divided into high- or low-score groups based on the best cutoff value determined by survminer package. The distribution of the survival time, survival status, ferroptosis scores, and expression profiles of three ferroptosis regulators is displayed in (Figure. 5A-B). The results showed that SLC7A1 and FANCD2 were overexpressed in the high-score group, while ACSL4 was up-regulated in the low-score group. Moreover, the ferroptosis score was positively correlated with the OS and PFS of ACC patients (Figure. 5C-D). Interestingly, we found that the ferroptosis scoring system can predict the OS and PFS of ACC patients who received mitotane therapy (Figure. 5E-F). Univariate and multivariate Cox regression analyses indicated that the ferroptosis score system could serve as an independent predictor for predicting the prognosis of ACC patients (Figure. $5 \mathrm{G}-\mathrm{H})$. Moreover, the score was associated with the stage $(\mathrm{P}=0.004), \mathrm{T}$ $(P=0.017)$, and metastasis status $(P=0.039)$ of ACC (Figure. 5I-K).

\subsection{Ferroptosis regulators related scoring system combined with immune factors predict prognosis and anti-tumor immune response in ACC}

Previous studies have demonstrated that TMB, PD-1, PD-L1, CTLA4, and immune cell infiltration not only related to the prognosis of cancers but also served as anti-tumor immune response biomarkers.

Therefore, we analyzed the correlation between ferroptosis regulators and these immune factors in ACC. The results showed that the ferroptosis score was positively related to the expression of PD-L1 and CTLA4 and was negatively related to the expression of TMB (Figure. 6A-C). However, the ferroptosis score was not associated with the expression of PD-1 (Figure. 6D ). Prognostic analyses showed that OS and PFS have significant advantages in patients with low TMB and PD-1, high expression of PD-L1 and CTLA4 (Figure. 6E-L). Further analyses suggested that the ferroptosis score integrated with various 
immune factors including CTLA4, PD-1, TMB, and PD-L1 expression, could predict the prognosis of ACC (Figure. 6I-P).

Additionally, the ferroptosis was significantly correlated with the immune cell infiltration in ACC by analyzing six immune cell infiltration datasets (Table 2). Among these immune cells, ferroptosis score and CD4+ $T$ cell infiltration exhibited a strong correlation in the tumor microenvironment of ACC (Figure. 7A-D). The infiltration of CD4+ T cells in the tumor microenvironment is also a favorable factor for predicting the prognosis of ACC (Figure. 7E-L). Further analyses suggested that the ferroptosis score integrated with $\mathrm{CD} 4+\mathrm{T}$ cell infiltration could predict the prognosis of ACC (Figure. 7M-T). These results indicated that the ferroptosis regulators related scoring system combined with immune factors not only more precisely predict prognosis but contribute to predicting anti-tumor immune response in ACC. 
Table 2

The correlation between ferroptosis score and immune cell infiltration in the microenvironment of ACC.

\begin{tabular}{|c|c|c|c|c|}
\hline $\begin{array}{l}\text { Immune } \\
\text { datasets }\end{array}$ & Ferroptosis score vs. & $\begin{array}{l}\text { Pearson } \\
r\end{array}$ & $95 \% \mathrm{Cl}$ & $\begin{array}{l}P \text { (two- } \\
\text { tailed) }\end{array}$ \\
\hline \multirow[t]{9}{*}{ ssGSEA } & Activated B cell & 0.1984 & 0.02509 to 0.3602 & 0.0253 \\
\hline & Activated CD4 T cell & -0.2043 & -0.3655 to -0.03119 & 0.0212 \\
\hline & Activated CD8 T cell & 0.2474 & 0.07644 to 0.4042 & 0.0051 \\
\hline & Activated dendritic cell & 0.2333 & 0.06155 to 0.3916 & 0.0083 \\
\hline & CD56bright natural killer cell & 0.2841 & 0.1156 to 0.4367 & 0.0012 \\
\hline & CD56dim natural killer cell & 0.3298 & 0.1651 to 0.4766 & 0.0002 \\
\hline & Eosinophil & 0.3299 & 0.1652 to 0.4767 & 0.0002 \\
\hline & Gamma delta T cell & 0.2944 & 0.1266 to 0.4457 & 0.0008 \\
\hline & Immature B cell & -0.4557 & -0.5836 to -0.3057 & $<0.0001$ \\
\hline \multirow[t]{5}{*}{ CIBERSORT } & $\mathrm{B}$ cell naive & -0.2406 & -0.4421 to -0.01604 & 0.0363 \\
\hline & B cell plasma & -0.2335 & $\begin{array}{l}-0.4360 \text { to } \\
-0.008496\end{array}$ & 0.0424 \\
\hline & CD4+ memory resting $\mathrm{T}$ cell & 0.4126 & 0.2063 to 0.5837 & 0.0002 \\
\hline & follicular helper T cell & -0.3083 & -0.4990 to -0.08899 & 0.0067 \\
\hline & M0 Macrophage & -0.2432 & -0.4443 to -0.01873 & 0.0343 \\
\hline \multirow[t]{4}{*}{ QUANTISEQ } & B cell & -0.4927 & -0.6463 to -0.3006 & $<0.0001$ \\
\hline & M2 Macrophage & 0.335 & 0.1185 to 0.5211 & 0.0031 \\
\hline & CD4+ T cell & 0.4476 & 0.2471 to 0.6114 & $<0.0001$ \\
\hline & Myeloid dendritic cell & -0.3146 & -0.5043 to -0.09591 & 0.0056 \\
\hline \multirow[t]{2}{*}{ MCPCOUNTER } & $\mathrm{T}$ cell & -0.2374 & -0.4393 to -0.01261 & 0.0389 \\
\hline & Myeloid dendritic cell & 0.2926 & 0.07189 to 0.4860 & 0.0103 \\
\hline \multirow[t]{4}{*}{ XCELL } & CD4+ memory T cell & 0.2369 & 0.01207 to 0.4389 & 0.0394 \\
\hline & CD4+ naive $\mathrm{T}$ cell & 0.2304 & 0.005248 to 0.4334 & 0.0452 \\
\hline & CD4+ central memory $\mathrm{T}$ cell & -0.423 & -0.5920 to -0.2184 & 0.0001 \\
\hline & CD4+ effector memory T cell & 0.2282 & 0.002854 to 0.4314 & 0.0474 \\
\hline
\end{tabular}

$\mathrm{Cl}$, confidence interval. 


\begin{tabular}{|lllll|}
\hline $\begin{array}{l}\text { Immune } \\
\text { datasets }\end{array}$ & Ferroptosis score vs. & $\begin{array}{l}\text { Pearson } \\
\mathbf{r}\end{array}$ & $\mathbf{9 5 \%} \mathrm{Cl}$ & $\begin{array}{l}\text { P (two- } \\
\text { tailed) }\end{array}$ \\
\hline $\begin{array}{l}\text { Class-switched memory B } \\
\text { cell }\end{array}$ & -0.2273 & $\begin{array}{l}-0.4306 \text { to } \\
-0.001896\end{array}$ & 0.0484 \\
\hline M2Macrophage & 0.342 & 0.1263 to 0.5269 & 0.0025 \\
\hline NK T cell & 0.3919 & 0.1826 to 0.5672 & 0.0005 \\
\hline CD4+ Th1 T cell & -0.2598 & -0.4584 to -0.03647 & 0.0234 \\
\hline CD4+ Th2 T cell & -0.2522 & -0.4520 to -0.02836 & 0.028 \\
\hline regulatory T cell (Tregs) & 0.2806 & 0.05892 to 0.4760 & 0.0141 \\
\hline CPIC & 0.2952 & 0.07468 to 0.4881 & 0.0096 \\
\hline Cl, confidence interval. & & & \\
\hline
\end{tabular}

\subsection{Construction of a prognostic nomogram for ACC}

To establish a clinically applicable method for monitoring the prognosis of ACC patients, we construct a prognostic nomogram by using clinical characteristics (age, gender, stage, $T, N, M$, invasion of tumor capsule, mitotic rate $>5 / 50$ HPF, Nuclear grade III or IV, Weiss score, radiation therapy, and mitotane therapy, and), TMB, PD-L1 expression, CTLA4 expression, PD-1 expression, CD4+ T cell infiltration, and the ferroptosis score. The result indicated that the new prognostic nomogram could superiorly predict the 1-, 2-, 3, 5-year OS and PFS of ACC patients (Figure. 8A-B). The calibration plots also validate excellent agreement between prediction and observation for the 3- and 5-year OS and PFS probabilities of ACC patients (Figure. 8C-F).

\subsection{Validation of the expression levels of three ferroptosis regulators}

RNA isolation and reverse transcription-quantitative PCR (RT-qPCR) was further performed to validate the expression levels of the three selected ferroptosis regulators in human ACC tissues and normal tissues. The results demonstrated significant differences in the expression levels of ACSL4 (A-D) FANCD2 (E-H) and SLC7A1 (I-L) between ACC and normal tissues. Compared to normal tissues, three ferroptosis regulators were up-regulated in ACC (Figure. 9).

\section{Discussion}

ACC is a rare endocrine malignant tumor with a high risk of metastasis, few prognostic predictors, and limited treatment options. To improve ACC prognosis and treatment, identify powerful prognostic biomarkers and novel therapeutic strategies are urgent needs. Ferroptosis, a programmed necrosis factor, plays a vital role in killing cancer cells and inhibiting cancer growth by mediating multiple biological 
pathways, including P53 and Ras-MEK pathways [15]. However, the role of ferroptosis regulators in the malignancy prognosis, immune cell infiltration of ACC has not been comprehensively investigated.

In the present study, the expression profiles and prognostic values of ferroptosis regulators in ACC were first demonstrated. Most of the regulators were differentially expressed in ACC and were significantly related to the prognosis and clinical characteristics of ACC. The results indicated that the expression imbalance of ferroptosis regulators possessed critical biological roles in the tumorigenesis and progression of ACC. To explore the biological functions of ferroptosis regulators in ACC, consensus clustering was performed to divided ACC into different subgroups based on the expression similarity of ferroptosis regulators. We revealed three ferroptosis subgroups (Cluster A, B, C), which have significantly distinct survival outcomes in ACC. Among three groups, patients in Cluster C had longer OS and PFS, which indicated ferroptosis regulators were associated with the heterogeneity of ACC. To explain the phenomenon, a GSVA enrichment analysis was performed. Interestingly, we found that Cluster $\mathrm{C}$ was prominently related to immune fully activation including chemokine signaling pathway, natural killer cellmediated cytotoxicity, $T$ cell receptor signaling pathway, and Toll-like receptor signaling pathway. These pathways were contributed to the anti-tumor immune response against numerous malignancies [16-18]. Subsequently, the immune cell infiltration and antigen-presenting genes expression in different subgroups were investigated. The results also showed that multiple immune cells and antigen-presenting genes were highly expressed in Cluster C, representing the strong immunogenicity of Cluster C in ACC. Overall, ferroptosis regulators were closely related to the prognosis, immune microenvironment remodeling, and anti-tumor immune response of ACC.

Considering the individual heterogeneity of ferroptosis regulators, we constructed a scoring system to assess the ferroptosis modification pattern of individual ACC patients based on the expression of SC7A1, FANCD2, and ACSL4. Survival analyses showed that the ferroptosis score not only independently predict the prognosis of ACC, but also could predict the survival time of ACC patients who received mitotane therapy. This suggested that ferroptosis regulators could be used to further predict the efficiency of mitotane. Previous results showed that ferroptosis regulators were of great significance in anti-tumor immune response and shaping different tumor immune microenvironments. Moreover, the mediation of ferroptosis regulators on tumor immune cell infiltration and immune-related genes is still limited.

Therefore, it was urgently needed to analyze the relationship between ferroptosis score and immune factors in ACC. PD-1, PD-L1, and TMB were the main potential markers of response to immunotherapy in ACC [19]. Our study showed that ferroptosis scores were positively related to the expression of PD-L1 and were negatively related to the expression of TMB. However, the ferroptosis score was not associated with the expression of PD-1. Furthermore, ferroptosis scores were correlated with the infiltration levels of multiple immune cells, especially CD4+ T cells. CD4+ T cells can target tumor cells in various ways, either directly by eliminating tumor cells through cytolytic mechanisms or indirectly by modulating the tumor microenvironment [20]. The above results implying ferroptosis regulators could influence the efficacy of immune checkpoint blockade and shape the immune tumor microenvironment landscape. The ferroptosis score integrated various immune biomarkers including TMB, PD-L1, PD-1, CTLA4, and CD4+ T cell could be the effective predictive strategies for the prognosis and immunotherapy of ACC. 
However, there are several limitations to our study. First, the expression levels of ferroptosis regulators needed to be further validated in ACC samples or ACC cells by immunohistochemical and Western blot. Secondly, the ferroptosis score and interaction between the immune factors and ferroptosis regulators should be subject to external verification in multicenter cohorts. In addition, the regulatory mechanisms of ferroptosis regulators in ACC are warranted to be further explored to remodel the immune microenvironment and improve the precision in immunotherapy of ACC.

\section{Conclusions}

In conclusion, our study demonstrated ferroptosis regulators were significantly associated with the prognosis, clinical characteristics, immune-checkpoint genes expression, and tumor microenvironment immune cell infiltration in ACC. This current study provided comprehensive evidence for further research on ferroptosis regulators in ACC and provides new enlightenment for epigenetic regulation of antitumor immune response.

\section{Abbreviations}

ACC, Adrenocortical carcinoma;

TCGA, The Cancer Genome Atlas;

GEO, Gene Expression Omnibus;

OS, Overall survival;

PFS, Progression free survival;

TMB, Tumor mutation burden;

GTEx, Genotype-Tissue Expression;

TIMER, Tumor Immune Estimation Resource;

PCA, Principal Component Analysis;

sSGSEA, single-sample gene-set enrichment analysis;

GSVA, Gene Set Variation Analysis;

PD-L1, programmed death-ligand 1;

CTLA4, cytotoxic T-lymphocyte associated protein 4;

PD-1, programmed death 1 


\section{Declarations}

\section{Funding statement}

This work was supported by the National Natural Science Foundation of China (Nos. 81972378, 81101932).

\section{Author Contributions Section}

Chengquan Shen: Conceptualization, Methodology, Data curation, and Writing - original draft. Jing Liu and Ye Liang: Validation. Zhijuan, Liang, Liping Wang, Yuanbin Chen, and Dan Li: Visualization, and Software. Yonghua Wang: Writing - review \& editing. All authors contributed to manuscript revision, read, and approved the submitted version.

\section{Availability of data and material}

The data used to support the findings of this study are included in the article, and the data are available from the corresponding author upon request.

\section{Acknowledgments}

None.

\section{Ethics approval and consent to participate}

Not applicable.

\section{Consent for publication}

Not applicable.

\section{Disclosure Statement}

The authors report no conflict of interest.

\section{References}

1. Crona J, Baudin E, Terzolo M, Chrisoulidou A, Angelousi A, Ronchi CL, Oliveira CL, Nieveen van Dijkum EJM, Ceccato F, Borson-Chazot F, Reimondo G, Tiberi GAM, Ettaieb H, Kiriakopoulos A, Canu L, Kastelan D, Osher E, Yiannakopoulou E, Arnaldi G, Assié G, Paiva I, Bourdeau I, Newell-Price J, Nowak KM, Romero MT, De Martino MC, Bugalho MJ, Sherlock M, Vantyghem MC, Dennedy MC, Loli P, Rodien P, Feelders R, de Krijger R, Van Slycke S, Aylwin S, Morelli V, Vroonen L, Shafigullina Z, Bancos I, Trofimiuk-Müldner M, Quinkler M, Luconi M, Kroiss M, Naruse M, Igaz P, Mihai R, Della Casa S, Berruti A, Fassnacht M, Beuschlein F. ENSAT registry-based randomized clinical trials for adrenocortical carcinoma. Eur J Endocrinol. 2021 Feb;184(2):R51-9. doi:10.1530/EJE-20-0800. 
2. Crona J, Beuschlein F. Adrenocortical carcinoma - towards genomics guided clinical care. Nat Rev Endocrinol. 2019 Sep;15(9):548-60. doi:10.1038/s41574-019-0221-7.

3. Stockwell BR, Friedmann Angeli JP, Bayir H, Bush Al, Conrad M, Dixon SJ, Fulda S, Gascón S, Hatzios SK, Kagan VE, Noel K, Jiang X, Linkermann A, Murphy ME, Overholtzer M, Oyagi A, Pagnussat GC, Park J, Ran Q, Rosenfeld CS, Salnikow K, Tang D, Torti FM, Torti SV, Toyokuni S, Woerpel KA, Zhang DD. Ferroptosis: A Regulated Cell Death Nexus Linking Metabolism, Redox Biology, and Disease. Cell. 2017 Oct 5;171(2):273-285. doi: 10.1016/j.cell.2017.09.021.

4. Dixon SJ. Ferroptosis: bug or feature? Immunol Rev. 2017 May;277(1):150-7. doi:10.1111/imr.12533.

5. Eling N, Reuter L, Hazin J, Hamacher-Brady A, Brady NR. Identification of artesunate as a specific activator of ferroptosis in pancreatic cancer cells. Oncoscience. 2015 May 2;2(5):517-32. doi: 10.18632/oncoscience.160.

6. Friedmann Angeli JP, Krysko DV, Conrad M. Ferroptosis at the crossroads of cancer-acquired drug resistance and immune evasion. Nat Rev Cancer. 2019 Jul;19(7):405-14. doi:10.1038/s41568-0190149-1.

7. Dai E, Han L, Liu J, Xie Y, Kroemer G, Klionsky DJ, Zeh HJ, Kang R, Wang J, Tang D. Autophagydependent ferroptosis drives tumor-associated macrophage polarization via release and uptake of oncogenic KRAS protein. Autophagy. 2020 Nov;16(11):2069-83.

doi:10.1080/15548627.2020.1714209.

8. Wang W, Green M, Choi JE, Gijón M, Kennedy PD, Johnson JK, Liao P, Lang X, Kryczek I, Sell A, Xia H, Zhou J, Li G, Li J, Li W, Wei S, Vatan L, Zhang H, Szeliga W, Gu W, Liu R, Lawrence TS, Lamb C, Tanno Y, Cieslik M, Stone E, Georgiou G, Chan TA, Chinnaiyan A, Zou W. CD8+ T cells regulate tumour ferroptosis during cancer immunotherapy. Nature. 2019 May;569(7755):270-4. doi:10.1038/s41586019-1170-y.

9. Tian X, Xu W, Wang Y, Anwaier A, Wang H, Wan F, Zhu Y, Cao D, Shi G, Zhu Y, Qu Y, Zhang H, Ye D. Identification of tumor-infiltrating immune cells and prognostic validation of tumor-infiltrating mast cells in adrenocortical carcinoma: results from bioinformatics and real-world data. Oncoimmunology. 2020 Jun 23;9(1):1784529. doi: 10.1080/2162402X.2020.1784529.

10. Brabo EP, Moraes AB, Neto LV. The role of immune checkpoint inhibitor therapy in advanced adrenocortical carcinoma revisited: review of literature. J Endocrinol Invest. 2020 Nov;43(11):153142. doi:10.1007/s40618-020-01306-5.

11. Charoentong P, Finotello F, Angelova M, Mayer C, Efremova M, Rieder D, Hackl H, Trajanoski Z. Pancancer Immunogenomic Analyses Reveal Genotype-Immunophenotype Relationships and Predictors of Response to Checkpoint Blockade. Cell Rep. 2017 Jan 3;18(1):248-262. doi: 10.1016/j.celrep.2016.12.019.

12. Hänzelmann S, Castelo R, Guinney J. GSVA: gene set variation analysis for microarray and RNA-seq data. BMC Bioinformatics. 2013 Jan 16;14:7. doi: 10.1186/1471-2105-14-7. 
13. Zhang B, Wu Q, Li B, Wang D, Wang L, Zhou YL. m6A regulator-mediated methylation modification patterns and tumor microenvironment infiltration characterization in gastric cancer. Mol Cancer. 2020 Mar 12;19(1):53. doi: 10.1186/s12943-020-01170-0.

14. Shen C, Liu J, Yang X, Jiao W, Wang Y. Development and Validation of an m6A RNA Methylation Regulators-Based Signature for Predicting the Prognosis of Adrenocortical Carcinoma. Front Endocrinol (Lausanne). 2021 Feb;22:12:568397. doi:10.3389/fendo.2021.568397.

15. Mou Y, Wang J, Wu J, He D, Zhang C, Duan C, Li B. Ferroptosis, a new form of cell death: opportunities and challenges in cancer. J Hematol Oncol. 2019 Mar 29;12(1):34. doi: 10.1186/s13045-019-0720-y.

16. Romanski A, Bug G, Becker S, Kampfmann M, Seifried E, Hoelzer D, Ottmann OG, Tonn T. Mechanisms of resistance to natural killer cell-mediated cytotoxicity in acute lymphoblastic leukemia. Exp Hematol. 2005 Mar;33(3):344-52. doi: 10.1016/j.exphem.2004.11.006.

17. Alcover A, Alarcón B, Di Bartolo V. Cell Biology of T Cell Receptor Expression and Regulation. Annu Rev Immunol. 2018 Apr;26:36:103-25. doi:10.1146/annurev-immunol-042617-053429.

18. Moradi-Marjaneh R, Hassanian SM, Fiuji H, Soleimanpour S, Ferns GA, Avan A, Khazaei M. Toll like receptor signaling pathway as a potential therapeutic target in colorectal cancer. J Cell Physiol. 2018 Aug;233(8):5613-22. doi:10.1002/jcp.26273.

19. Araujo-Castro M, Pascual-Corrales E, Molina-Cerrillo J, Alonso-Gordoa T. Immunotherapy in Adrenocortical Carcinoma: Predictors of Response, Efficacy, Safety, and Mechanisms of Resistance. Biomedicines. 2021 Mar 16;9(3):304. doi: 10.3390/biomedicines9030304.

20. Borst J, Ahrends T, Bąbała N, Melief CJM, Kastenmüller W. CD4+ T cell help in cancer immunology and immunotherapy. Nat Rev Immunol. 2018 Oct;18(10):635-47. doi:10.1038/s41577-018-0044-0.

\section{Figures}



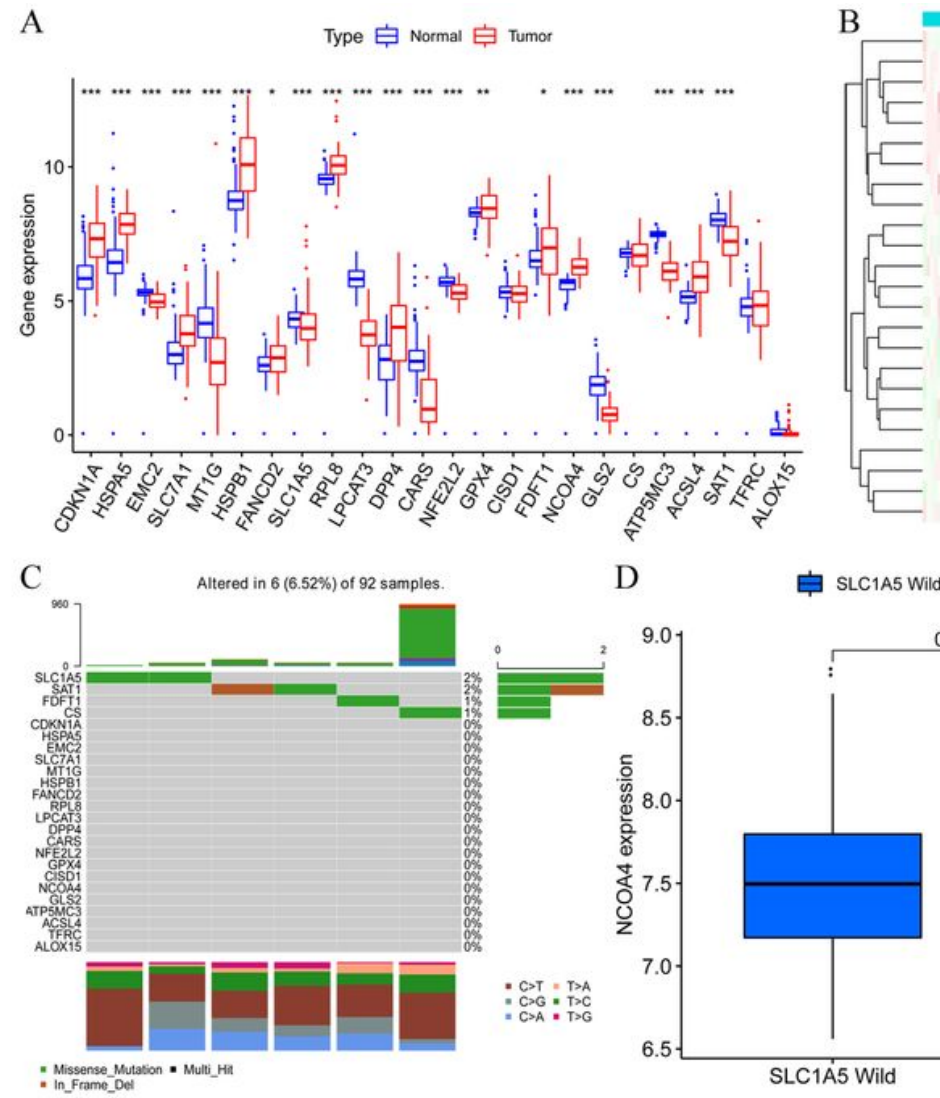

D
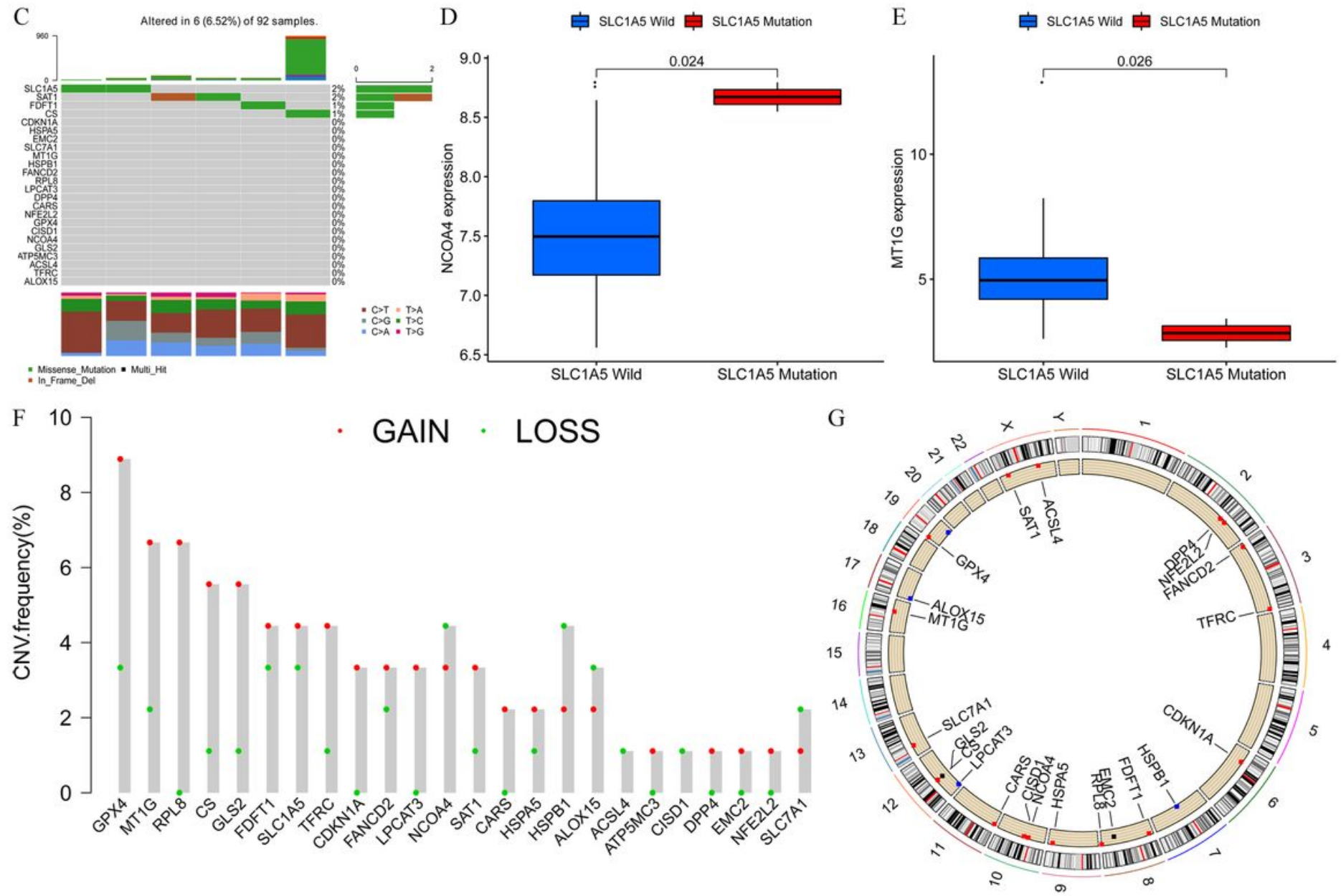

\section{Figure 1}

Landscape of genetic variation of ferroptosis regulators in ACC. (A-B) The expression profiles of ferroptosis regulators between ACC and normal samples. Tumor, red; blue, normal. The asterisks represented the statistical $p$-value ( $\left.{ }^{\star} P<0.05 ; * \star P<0.01 ; \star \star \star P<0.001\right)$. (C) The mutation frequency of 21 ferroptosis regulators in 92 ACC patients. (D-E) Compared with SLC1A5 wild types, NCOA4 and MT1G were differentially expressed in SLC1A5 mutant types. (F) The CNV variation frequency of ferroptosis regulators in ACC. The height of the column represented the alteration frequency. The blue dot represented the deletion frequency and the red dot represented amplification frequency. (G) The location of CNV alteration of ferroptosis regulators on 23 chromosomes. 


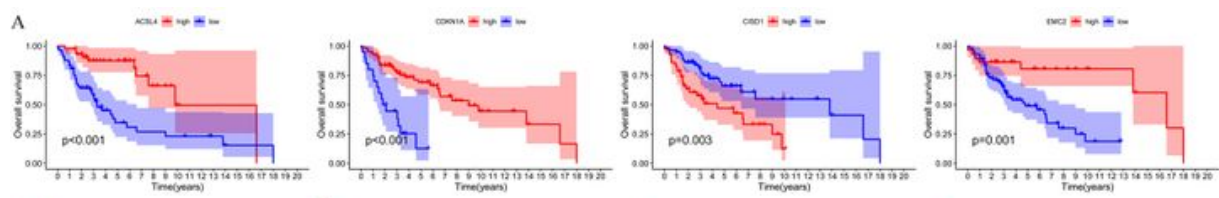
要

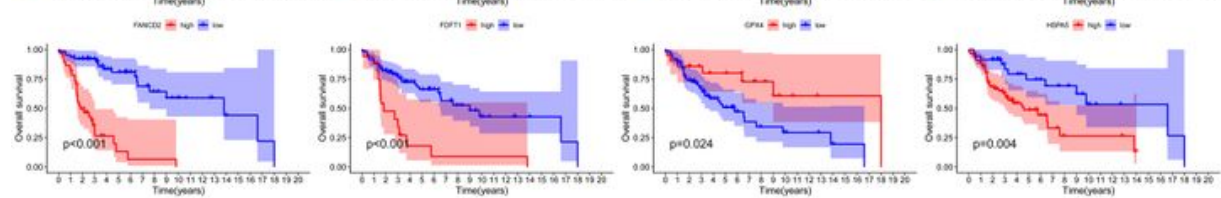

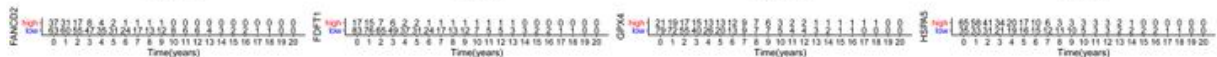

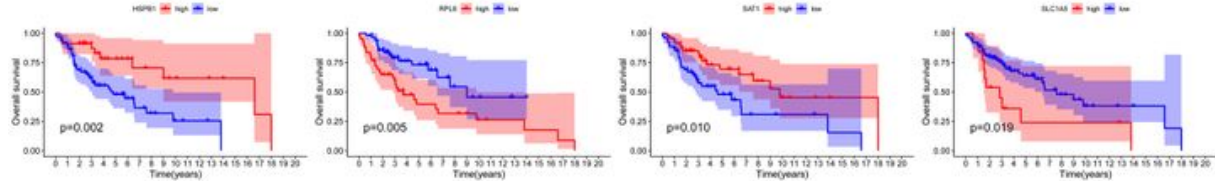

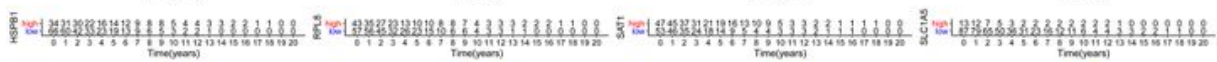

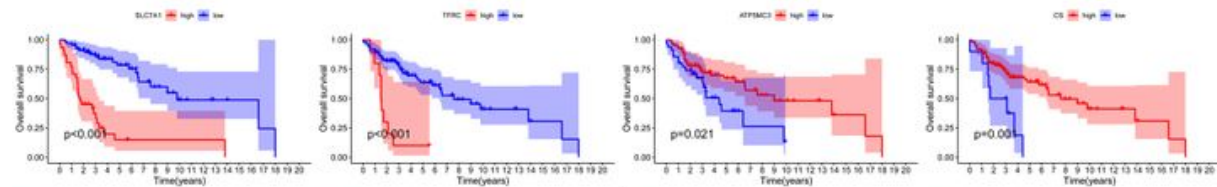

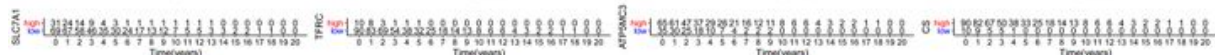

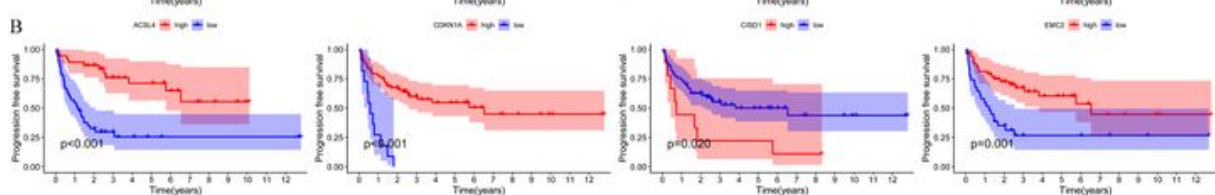

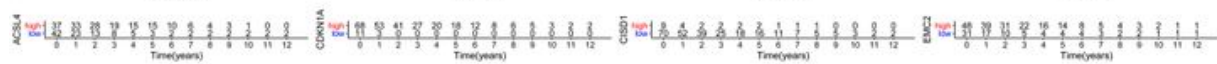

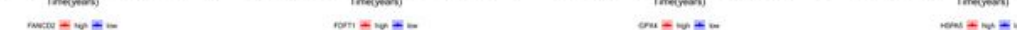

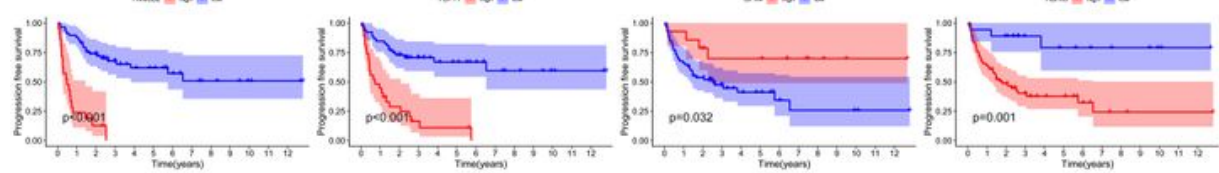

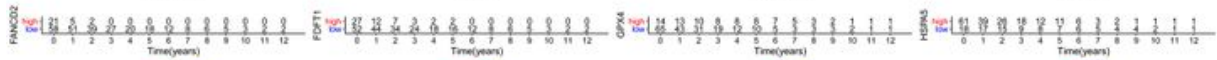
M-
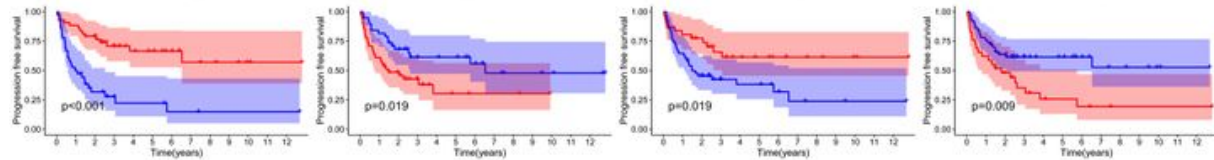

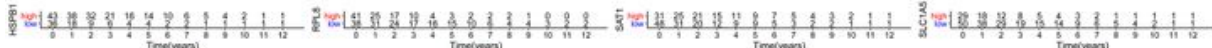

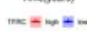
ast $=-\infty=-$ $\cos x \rightarrow \infty x+$
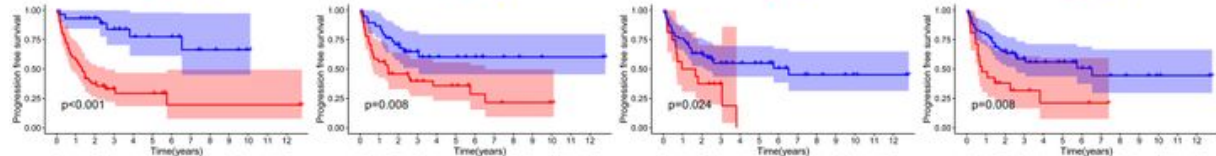

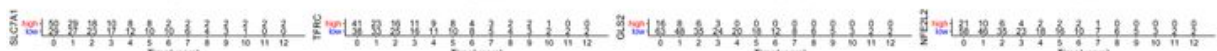

\section{Figure 2}

Prognostic values of ferroptosis regulators in ACC. (A) Twelve ferroptosis regulators were related to the overall survival of ACC. (B) Twelve ferroptosis regulators were related to the progression free survival of ACC. 

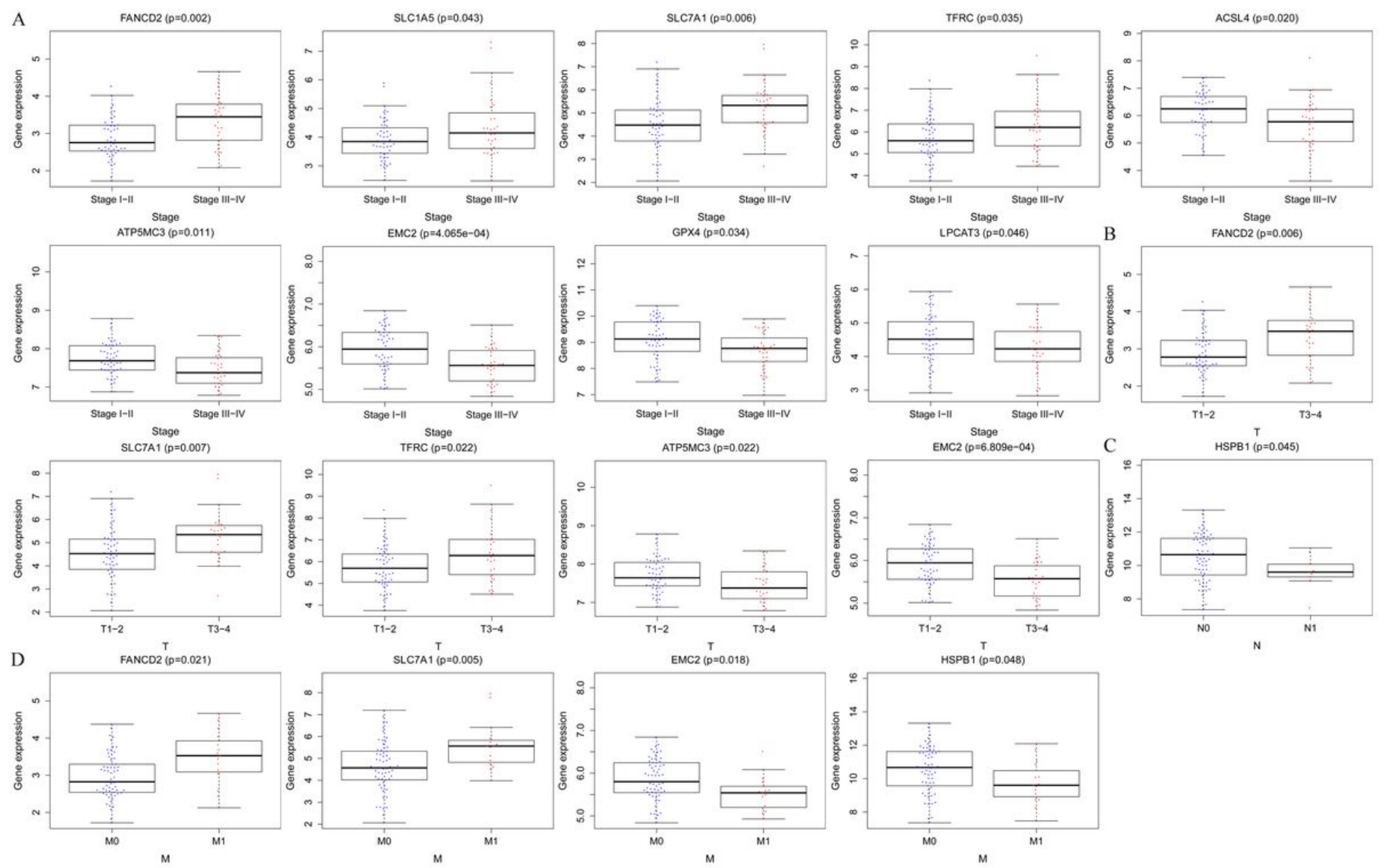

\section{Figure 3}

The correlation between ferroptosis regulators and clinical characteristics in ACC. (A) The high stage had higher expression of FANCD2, SLC1A5, SLC7A1, and TFRC, but lower expression of ACSL4, ATP5MC3, EMC2, GPX4, and LPCAT3. (B) FANCD2, SLC7A1, and TFRC have high expression, while ATP5CM3 and EMC2 have low expression in ACC patients with advanced T. (C) Compared to ACC patients without lymph node metastasis, HSPB1 down-regulated in those with lymph node metastasis. (D) FANCD2 and SLC7A1 may promote the metastasis of ACC, while EMC2 and HSPB1 may inhibit the metastasis of ACC. 

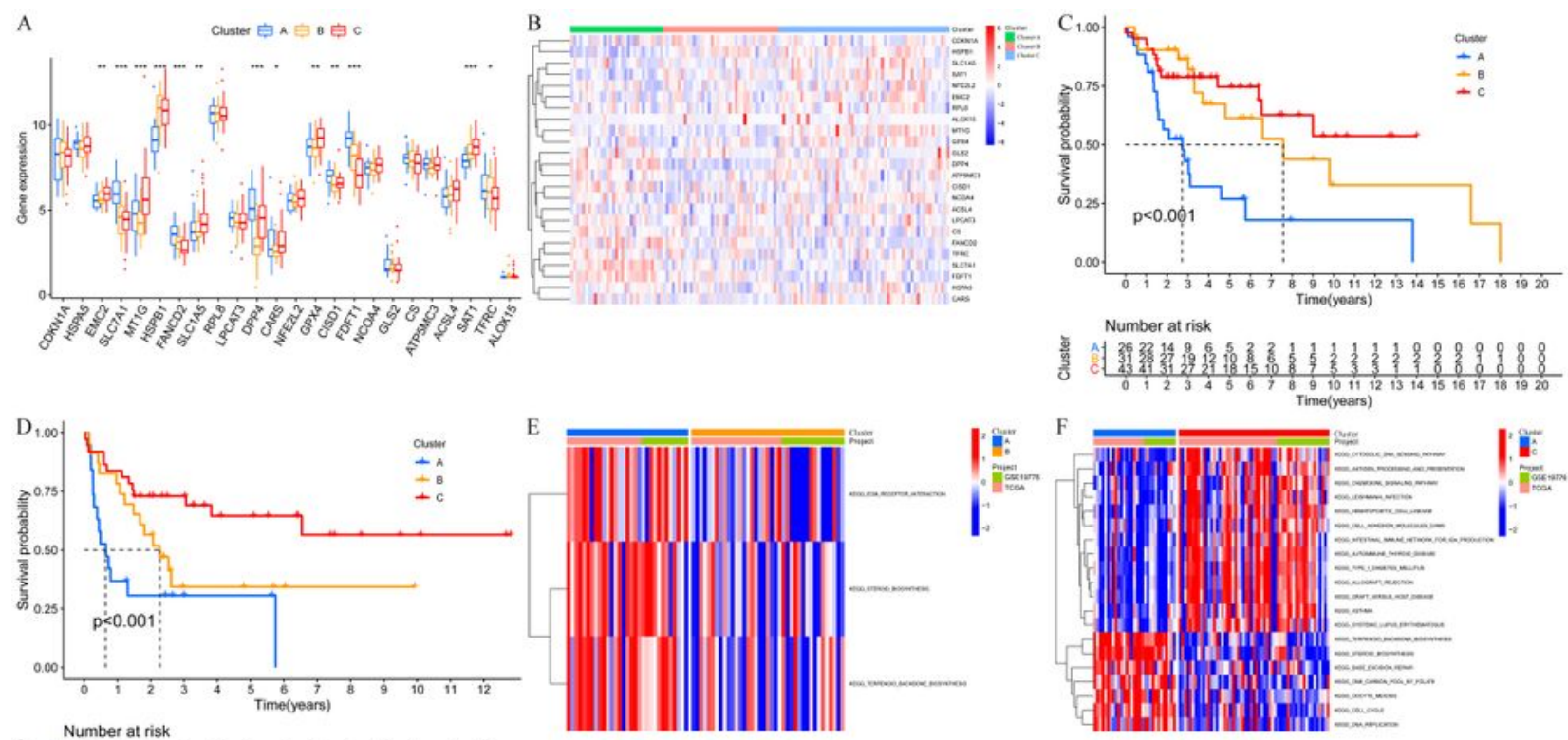

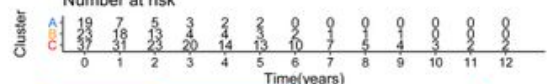
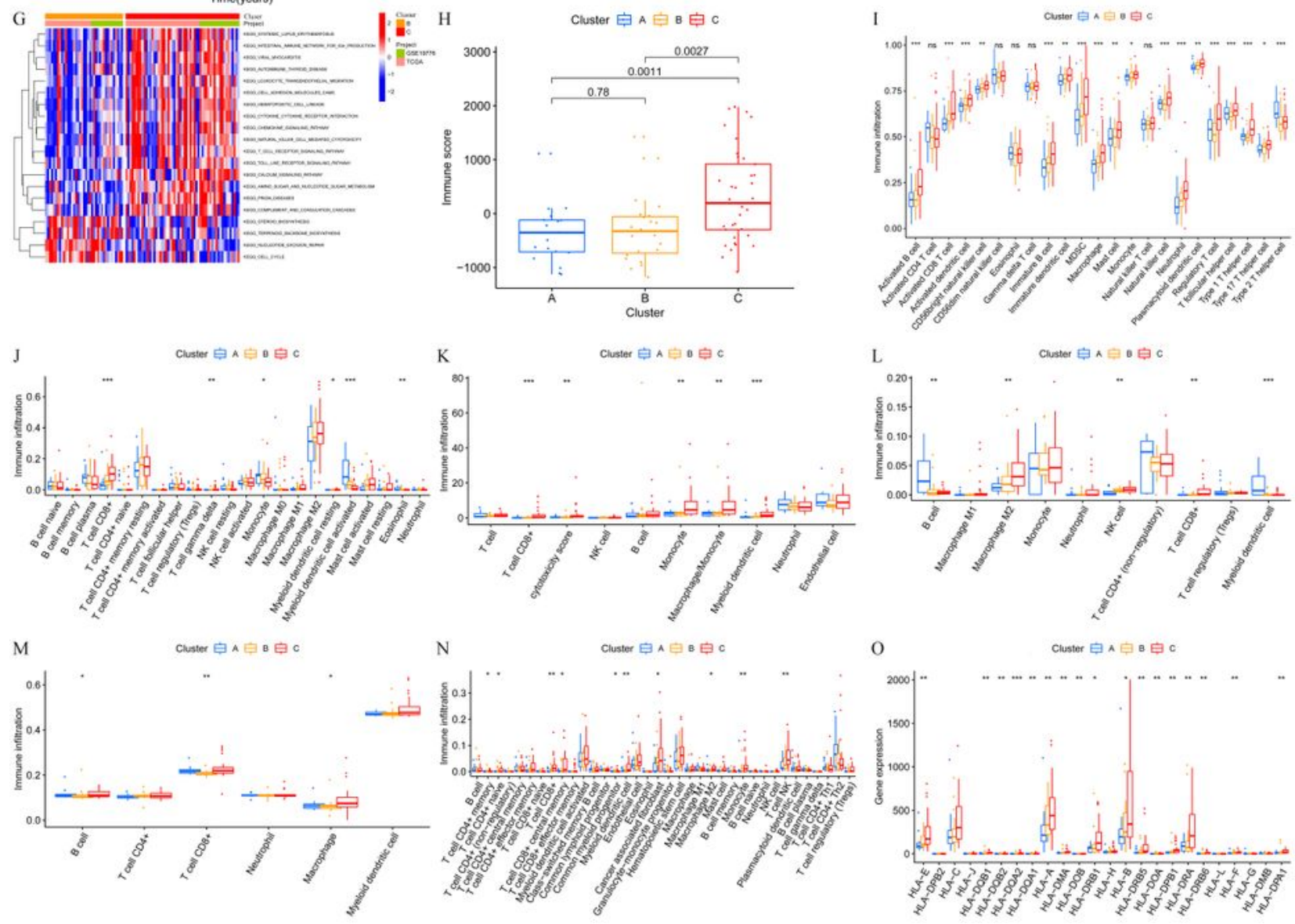

\section{Figure 4}

Correlation of ferroptosis clusters with prognosis, biological pathways, and immune cell infiltration. (A-B) The expression levels of ferroptosis regulator in three clusters. The asterisks represented the statistical $\mathrm{p}$ value ( $\left.{ }^{\star} P<0.05 ;{ }^{* *} P<0.01 ;{ }^{* * *} P<0.001\right)$. (C-D) Overall survival and progression free survival for three clusters. (E-G) GSVA enrichment analysis showing the activation states of biological pathways in three clusters. The heatmap was performed to visualize these biological pathways, and red represented 
activated pathways and blue represented inhibited pathways. $(\mathrm{H})$ Differences in immune score among three clusters in ACC. (I-N) Analysis of the infiltrating levels of immune cells in three clusters by using six datasets. I, ssGSEA; J CIBERSORT; K, MCPCOUNTER; L, QUANTISEQ; M, TIMER, N, XCELL. The upper and lower ends of the boxes represented the interquartile range of values. The lines in the boxes represented median value, and black dots showed outliers. The asterisks represented the statistical $p$-value $\left({ }^{\star} P<0.05\right.$; $\left.{ }^{* * P}<0.01 ; * \star * P<0.001\right) .(0)$ The expression levels of antigen-presenting genes in three clusters $\left({ }^{*} \mathrm{P}<\right.$ $0.05 ; * * \mathrm{P}<0.01 ; * \star * \mathrm{P}<0.001)$.
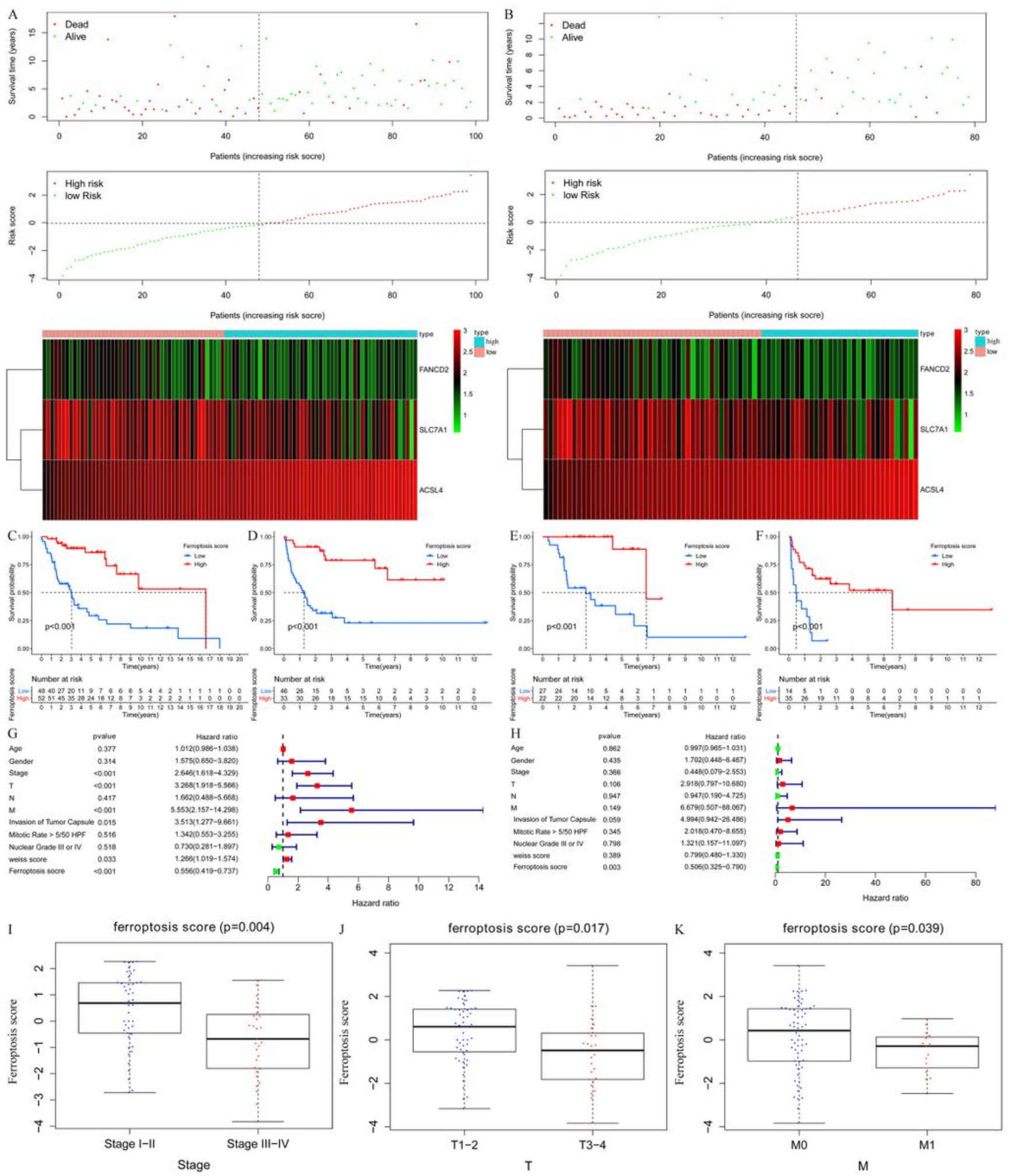


\section{Figure 5}

Generation of a ferroptosis regulators related scoring system. (A-B) The distribution of the survival time, survival status, ferroptosis scores, and expression profiles of three ferroptosis regulators. A, overall survival; B, progression survival. (C-D) Kaplan-Meier curves of overall survival and progression free survival for ACC patients based on the ferroptosis score. (E-F) Kaplan-Meier curves of overall survival and progression free survival for ACC patients who received mitotane therapy. (G-H) Univariate and multivariate Cox regression analyses indicated the ferroptosis score could independently predict the prognosis of ACC. (I-K) The ferroptosis score was associated with the stage, $\mathrm{T}$, and metastasis status of ACC. 

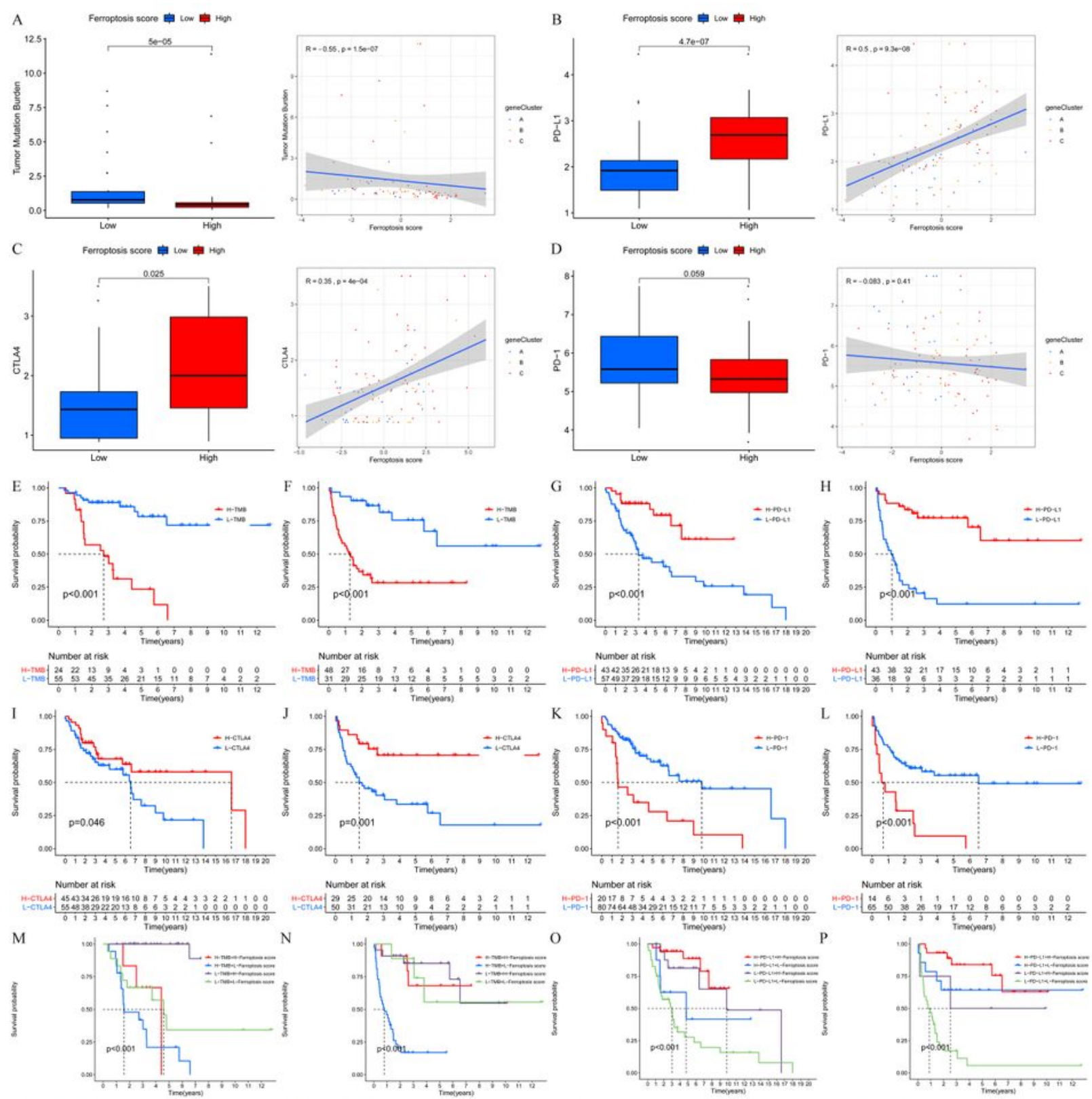

Number at risk Number at risk

Number at risk
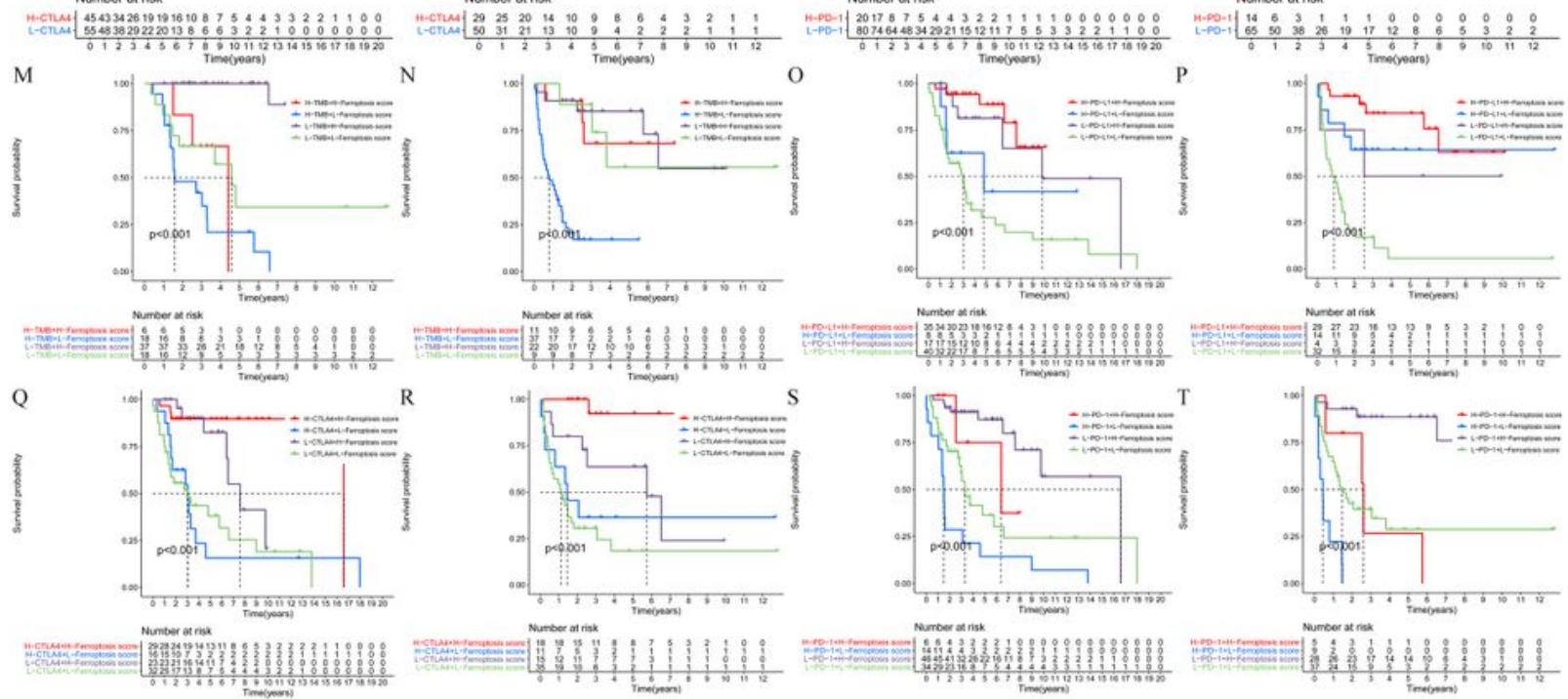

\section{Figure 6}

Ferroptosis score combined with immune factors predict prognosis and anti-tumor immune response in ACC. (A-D) The correlation between ferroptosis score and TMB, PD-L1 expression, CTLA4 expression, and PD-1 expression. TMB, tumor mutation burden; PD-L1, programmed death-ligand 1; CTLA4, cytotoxic Tlymphocyte associated protein 4, PD-1, programmed death 1. (E-L) Survival analyses for TMB, PD-L1, PD1, and CTLA4 in ACC. E, G, I, and K, overall survival; F, H, J, and L, progression free survival. (M-T) The 
ferroptosis score integrated with various immune factors including CTLA4, PD-1, TMB, and PD-L1 expression, could predict the prognosis of ACC.

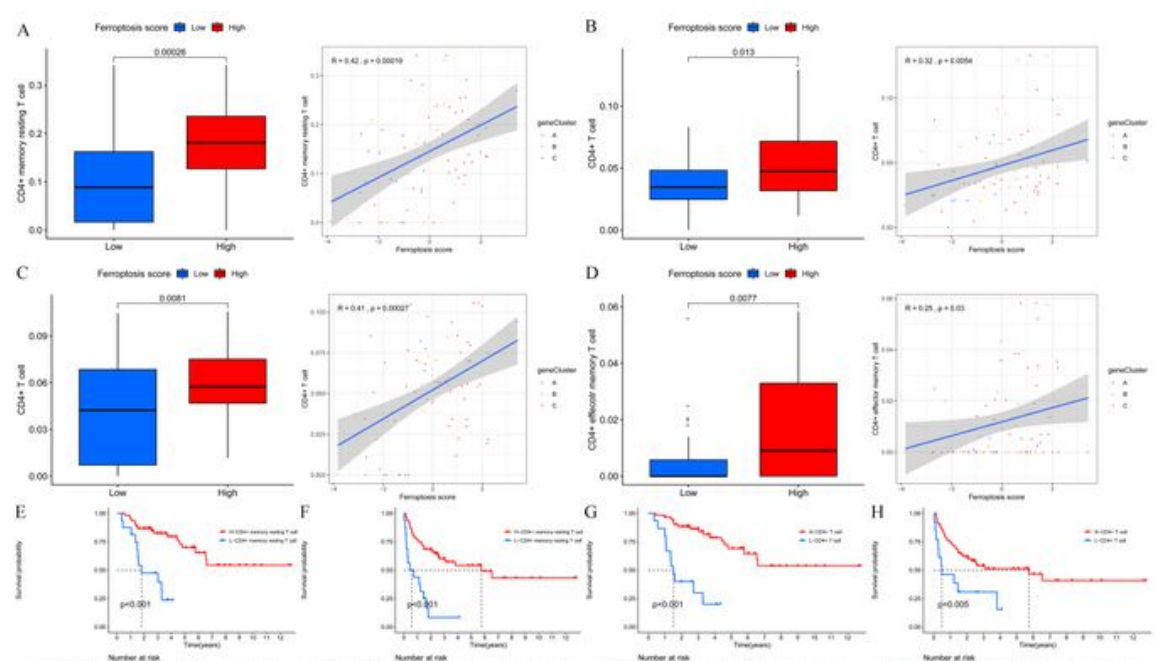

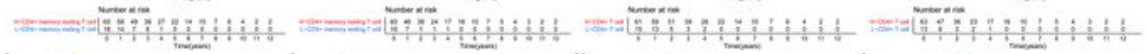

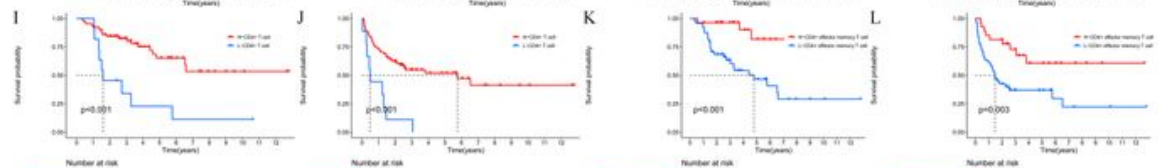

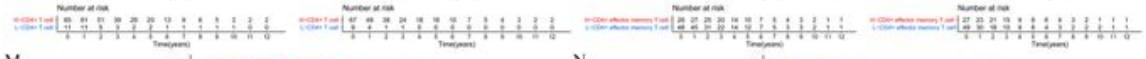
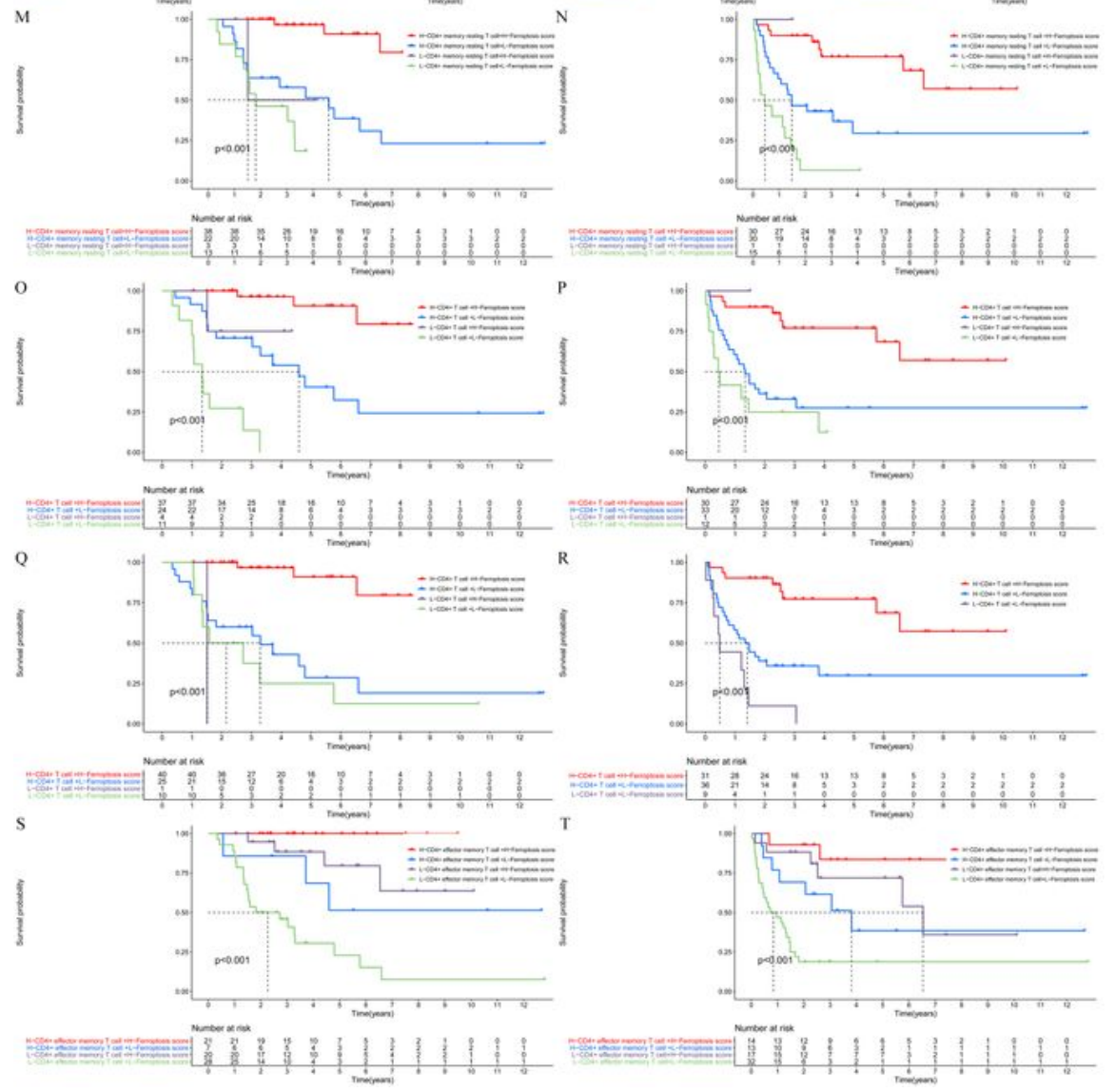

Figure 7 
Correlation analysis of CD4+ T cell infiltration with ferroptosis score and prognosis in ACC. (A-D) The correlation between ferroptosis score and CD4+ T cell infiltration in ACC. A, CIBERSORT; B, EPIC; C, QUANTISEQ; D, XCELL. (E-L) Survival analyses for CD4+ T cell in ACC. E, G, I, and K, overall survival; $F, H$, $J$, and L, progression free survival. E and F, CIBERSORT; $G$ and H, EPIC; I and J, QUANTISEQ; $K$ and $L$, XCELL. (M-T) The ferroptosis score integrated with CD4+ T cells could predict the prognosis of ACC. M, 0 , $Q$ and $S$, overall survival; $N, P, R$ and $T$, progression free survival. $M$ and $N, C$ CIBERSORT; $O$ and P, EPIC; $Q$ and R, QUANTISEQ; $S$ and T, XCELL. 

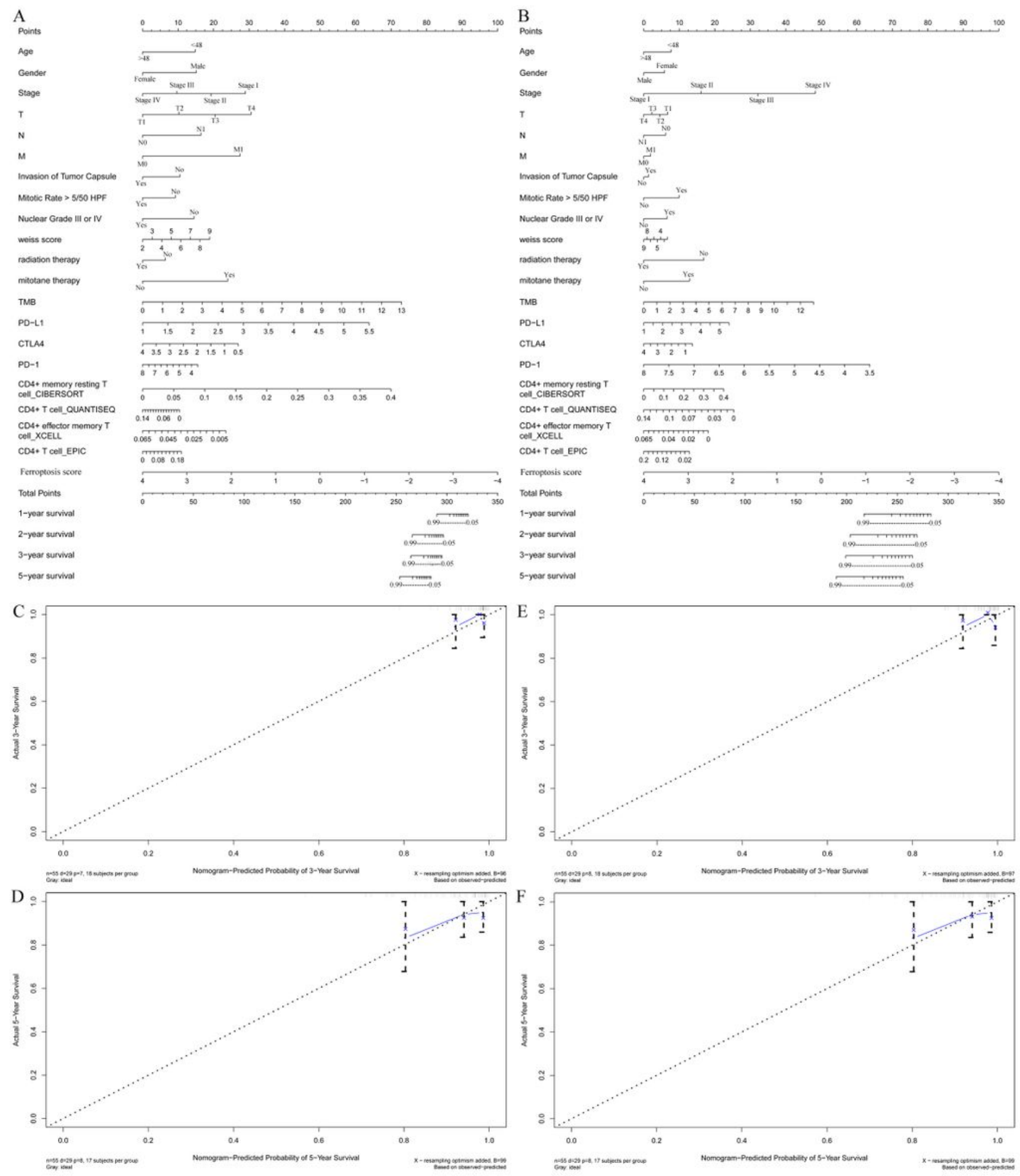

\section{Figure 8}

Construction of a nomogram to predict the prognosis of ACC. (A-B) The nomogram could superiorly predict 1-, 2-, and 3-, 5-year overall survival (OS) and progression free survival (PFS) of ACC patients. A, OS; B, PFS. (C-F) The calibration plots also demonstrate excellent agreement between prediction and observation for the 3 - and 5-year OS and PFS probabilities $(0.99,0.9,0.8,0.7,0.6,0.5,0.4,0.3,0.2,0.1$, 0.05) of the ACC patients. C and D, OS; E and F, PFS. 


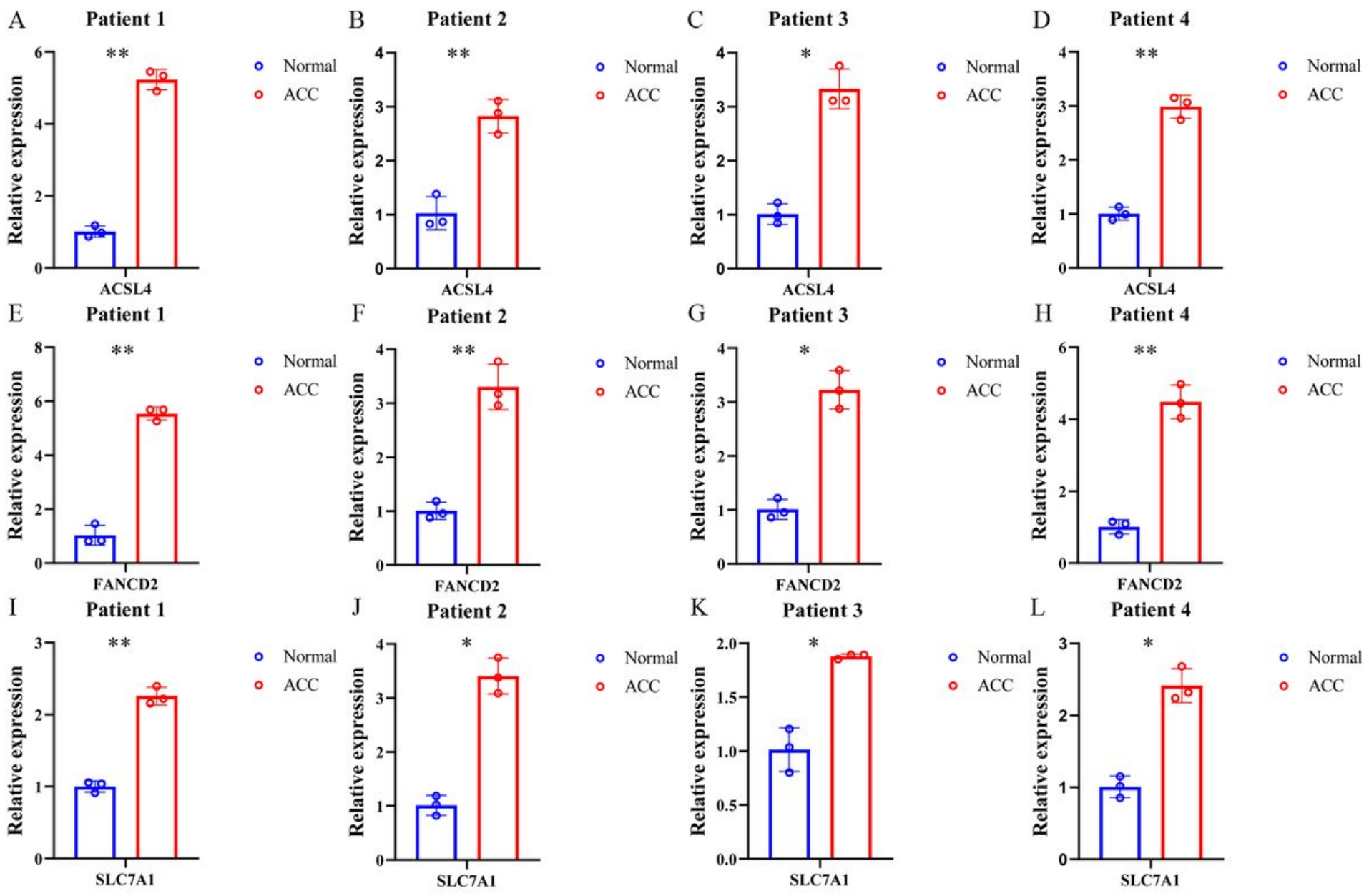

Figure 9

RT-PCR validation of three ferroptosis regulators in ACC and normal tissues. The results indicated that significant differences in the expression levels of ACSL4 (A-D) FANCD2 (E-H) and SLC7A1 (I-L) between ACC and normal tissues. ${ }^{*} \mathrm{p}<0.05 ;{ }^{* \star} \mathrm{p}<0.01 ;{ }^{\star \star *} \mathrm{p}<0.005$.

\section{Supplementary Files}

This is a list of supplementary files associated with this preprint. Click to download.

- Supplementary1.jpg

- Supplementary2.jpg

- Supplementary3.jpg 